\title{
As fast as it gets
}

Bart Verberck reflects on measuring the speed of light, its role in metrology, and special relativity.

or many, the speed of light in vacuum (c) probably brings to mind physics' most famous equation. For me, the power of $c$ is that, when postulated to be the same for different observers regardless of the motion of the light source, the special theory of relativity follows - which, also requiring the invariance of physics' laws in nonaccelerating frames of reference, of course gives you $E=m c^{2}$.

For metrologists, $c$ 's quintessential connotation is probably that of defining constant for the International System of Units (SI). Since 1983, the value of $c$ has been fixed at $299,792,458 \mathrm{~m} \mathrm{~s}^{-1}$, which enables a definition of the metre relating to it. The main advantage of defining units through physical constants with assigned values is that such definitions can forever remain unchanged - it's the practical realizations that can evolve and improve over time. Yet any such constant fixing needs to be preceded by high-precision, stateof-the-art measurements of the constant in question in the existing metrological framework. Only then can continuous and backward consistency be guaranteed.

Until very recently, $c$ was one of only two defining constants for the SI. But last month, during the 26th General Conference on Weights and Measures, fixed values for the Planck constant, the elementary charge, the Boltzmann constant and the Avogadro constant, and together with it the 'new SI', were ratified ${ }^{1}$, to become effective on 20 May 2019 - World Metrology Day.

One of the first attempts to measure light's speed goes back to Galileo Galilei; his experiment trying to establish whether there's a delay between uncovering a lit lantern and observing the emitted light at a distance was inconclusive, however.

Ole Rømer made an important step forward. Around 1676, he compared the timings of eclipses of Io, one of the moons of Jupiter, at different times throughout the year. The observed variation of the time intervals between eclipses was due to the changing Earth-Jupiter distance, he argued. Rømer estimated that to pass the Earth's orbital diameter, light travels for about
22 minutes. This insight led to a value of $220,000 \mathrm{~km} \mathrm{~s}^{-1}$ for $c$, based on a reasonable estimate for Earth's orbit ${ }^{2}$.

Proper terrestrial light-speed measurements were carried out around the middle of the nineteenth century by Hippolyte Fizeau. He let light reflect back to its source, with a rotating cogwheel put in the path of the double beam. When gradually increasing the rotational speed of the wheel, the situation becomes such that the incoming beam passes between the notch between two cogs and the reflected beam passes through the next notch. (In today's beam-scientist lingo: Fizeau used an optical chopper.) In 1849, he published a value of $315,000 \mathrm{~km} \mathrm{~s}^{-1}$ (ref. ${ }^{3}$ ).

Léon Foucault (of pendulum fame) developed a similar set-up. Instead of a cogwheel, he put a rapidly rotating mirror between the light source and the (now spherically curved) reflecting mirror. For large rotational speeds, a slit source is imaged at a small angle away from the source. The formula relating distance, angle and rotational speed allows a calculation of the speed of light. In 1862, he published the value $299,796 \mathrm{~km} \mathrm{~s}^{-1}$ (ref. ${ }^{4}$ ).

Both Fizeau's and Foucault's time-offlight experimental set-ups were refined in the following decades, leading to ever more accurate measurements of $c$. Albert Michelson made several measurements of $c$ between 1877 and his death in 1931, using adaptations of the Foucault apparatus allowing for longer distances and larger source-image angles. In 1930, he started performing Foucault-type experiments in a one-mile-long vacuum chamber (pictured). His work was continued posthumously by Fred Pease and Francis Pearson, who obtained the value $299,774 \pm 11 \mathrm{~km} \mathrm{~s}^{-1}$ (ref. ${ }^{5}$ ).

Alternative methods that provided better accuracy, developed in the following years, were based on cavity resonance or interferometry. By 1972, using laser interferometers, a fractional uncertainty of the order of $10^{-9}$ was achievable. Scientists at the National Bureau of Standards in the US achieved the result $c=299,792.4562 \pm$ $0.0011 \mathrm{~km} \mathrm{~s}^{-1}$ (ref. ${ }^{6}$ ). This, combined with

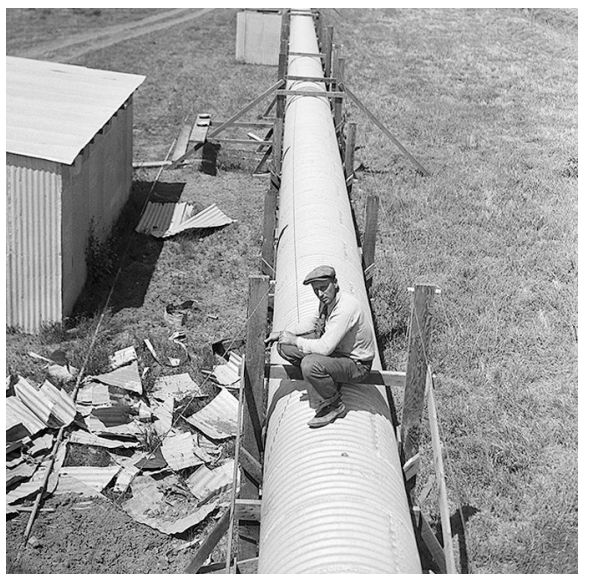

Credit: Bettmann/Getty Images

similar results, led to a recommendation of the 15th General Conference on Weights and Measures in 1975 of the value that would become the definition in 1983 .

Back to Albert Michelson, $c$ measurer par excellence. In 1887, together with Edward Morley, Michelson produced what is arguably the most famous null result of all time: a comparison of measurements of the speed of light in perpendicular directions revealed no difference, doing away with the thenprevalent concept of the 'aether', a medium pervading the cosmos believed necessary for transmitting forces. The Michelson-Morley result proved a stepping stone for Einstein to develop the theory of special relativity - and so physics' most famous equation.

\section{Bart Verberck \\ Regional executive editor for Nature Research. e-mail: bart.verberck@nature.com}

Published online: 4 December 2018 https://doi.org/10.1038/s41567-018-0374-7

References

1. NPL https://go.nature.com/2Bbf4EJ (2018).

2. Phil. Trans. R. Soc. 12, 893-95 (1677).

3. Fizeau, M. Comptes Rendus Acad. Sci. 29, 90-92 (1849).

4. Foucault, M. L. Comptes Rendus Acad. Sci. 55, 501-503 (1862).

5. Michelson, A. A., Pease, F. G. \& Pearson, F. Contrib. Mt Wilson Observ. 522, 1-36 (1935).

6. Evenson, K. M. et al. Phys. Rev. Lett. 29, 1346-1349 (1972).

$\mathrm{m} e \mathrm{~A} \mathrm{~s} \mathrm{u} R \mathrm{~s} \mathrm{E}_{\mathrm{h}} \mathrm{s} \mathrm{R} \mu_{0} \varepsilon_{0} \alpha \mathrm{R}$

